\title{
A SIMPLE BIO-SIGNALS QUALITY MEASURE FOR IN-HOME MONITORING
}

\author{
Kevin Sweeney \\ Department of Electronic Engineering \\ National University of Ireland Maynooth \\ Maynooth \\ Kildare, Ireland \\ email: ksweeney@eeng.nuim.ie
}

\author{
Seán McLoone and Tomás Ward \\ Department of Electronic Engineering \\ National University of Ireland Maynooth \\ Maynooth \\ Kildare, Ireland \\ email: sean.mcloone@eeng.nuim.ie \\ tomas.ward@eeng.nuim.ie
}

\begin{abstract}
Electrocardiography (ECG) is a test that measures the electrical activity of the heart. The use of ECG for recording in ambulatory settings is becoming more prominent due to an increase in in-home monitoring. By virtue of the ambulatory nature of the recordings, artifacts have a large effect on the signals, with the most significant artifact a result of motion. This paper describes an accelerometer system used to detect differential movement between the recording electrodes on the body. This system is then used to determine a Quality of Signal (QOS) metric for the ECG signal. The results show that the use of differential movement of the recording electrodes with respect to one another is a better representative of the motion artifact, then overall body movement.

This simple Signal Quality metric is used to more accurately flag the appropriate noisy ECG data which can be rejected from the signal. The simplicity of this system also allows it to be easily embedded into any in-home monitoring system.
\end{abstract}

\section{KEY WORDS}

Accelerometer, Quality of Signal, ECG, Motion Artifacts

\section{Introduction}

As life expectancy in the modern world continues to increase, the need for out-patient and in-home monitoring systems in the coming decades will rise considerably. This ability to monitor the patient, in the comfort of their own home, will greatly improve quality of life while increasing the efficiency of our healthcare system. As out-patient care continues to become more employed in the observation and treatment of patients, there is a corresponding increase in the need for more accurate ambulatory recordings of the physiological signals. These physiological signals include the heartbeat, brain signals, muscle signals and blood volume measured by Electrocardiography (ECG), Electroencephalography (EEG), Electromyography (EMG) and Photoplethysmography (PPG) respectively. However, a shift towards ambulatory recordings results in an increase in the interference embedded in the desired recorded physiolog- ical signal. Thus as the ambulatory physiological signal monitoring systems become more widespread, the need for systems and algorithms capable of removing these artifacts becomes fundamentally important.

Artifacts are defined as being any variations in a signal due to an unwanted external source. In ECG, artifact can come in two distinct forms; biological noise and environmental noise [1]. Biological noise is any signal that originates from the body, other then the desired signal. This can take the form of baseline wander $(<0.03 \mathrm{~Hz})$ due to respiration or the movement of the recording electrodes, high frequency noise $(1 \mathrm{~Hz}-5 \mathrm{kHz})$ due to EMG or muscle noise originating from the muscle movement within the human body and also motion artifact due to the movement of the human body and the displacement of the electrodes. Environmental interference originates from the surroundings of the subject. This can be seen as $(50 / 60 \mathrm{~Hz})$ interference from near-by power lines, high frequency noise from nearby instruments and electrode-skin contact noise. The artifacts which have the greatest impact on the ECG signal are motion artifact, baseline wander and power-line interference. As it is possible to remove both baseline wander and power-line interference from the desired signal by filtering, due to their narrow frequency bands, researchers are currently focused on motion artifacts. Motion artifact is the most difficult type of noise to detect since its spectrum completely overlaps that of the ECG and its morphology often resembles that of the $\mathrm{P}, \mathrm{QRS}$ and $\mathrm{T}$ waves (Figure 5) [2].

There have been many different methods undertaken to detect motion artifacts and remove them from desired signals. In 2008 it was shown that by providing a triaxial accelerometer as a reference to an adaptive filter the noise from an ECG signal could be reduced by up to $80 \%$ [1]. The majority of researchers have used accelerometers to detect the movement of the body, but other systems such as anisotropic magnetoresistive (AMR) sensors have also been considered [3]. In Mathie et al. [4] the author used a triaxial accelerometer to distinguish between states of rest and activity, thus proving that the decision to use accelerometers to detect body movement was a viable one. Raya and Sison [5] and Tong et al.[3] used single axis ac- 
celerometers to detect body movement in the lower back position and electrode placement position respectively. Using these accelerometer output signals as a reference for an adaptive filter the authors demonstrated the system effectively reduced the motion artifact in the recorded stress ECG.

Although these authors have succeeded in reducing the artifact embedded in stress ECG using adaptive filtering, an important question still stands as to the regions where these detected artifacts have the greatest impact in degrading the desired signal. A Quality of Signal (QOS) metric level can be attributed by knowing these positions and the level of impact that the motion artifact has on any particular portion of a signal. Therefore, each sample of the signal would have an individual confidence level attached to it. This QOS is then a help to technicians who are reading the ECG; with only a brief overview it is possible to detect the sections of the recorded ECG that are likely to be distorted by motion artifact.

As the vast majority of motion detection systems employ accelerometers, these are often used to apply a certain QOS level to the recorded ECG signal. In 2004, Muhlsteff et al. [6] tested a new design for a wearable ECG belt, with integrated dry electrodes. Although attempts to remove the motion artifacts using the accelerometer data failed, the accelerometer output was used to determine states of rest and activity of the subjects. By using these known states of activity, the author was able to apply a simple QOS metric and thus modify the levels of recorded, good, ECG detected. In 2005 Healey and Logan[7] used the accelerometer data in their "Wearable Wellness Monitoring System" to estimate the quality of the signal data and give a confidence rating on the results of their analysis algorithms. As the subjects activity level increased the quality of the ECG signal deteriorated, thus giving merit to their approach. Kishimoto et al. [8] recorded ECG in subjects as they slept. Any positional changes in the sleeping subject caused a disruption to the observed ECG. The authors therefore used an accelerometer attached to the body to detect when these body movements took place. They then used this detected movement as a QOS level for the recorded ECG stating that when positional changes occurred this resulted in a low QOS level.

Although it is known that movement of the body can have an adverse affect on the recorded ECG signal, this is not always the case. Over periods of large or constant movements, this paper will show that the ECG signal may not be consistently affected by the motion artifact. The papers cited above currently would determine that there is a low signal quality over the entire period of detected movement. For technicians viewing this ECG data with the corresponding QOS, a large amount of quality ECG may be discarded or ignored. In an ambulatory environment where, high quality, clean ECG is often difficult to record this loss of good data can be problematic. In this paper a method is proposed for quantifying the quality of the ECG signal by using a differential accelerometer system to detect movement of the recording electrodes on the body, with respect to one another, rather then the total body movement.

Section 2 of this paper describes our proposed system and the methodology of the conducted experiments, section 3 explains the findings of the tests and section 4 gives a brief overview of the findings of the paper.

\section{Methodology}

\subsection{Recording ECG}

Electrocardiography (ECG) is the recording of the electrical potential detected across the heart as it pumps blood around the bodies circulatory system. This recording process is usually performed non-invasively, with the electrodes connected to the skin of the patient using a conductive gel. The simplest implemented setup of ECG electrodes is the 3-Lead configuration. This specific setup measures the ECG from the right arm position to the left leg position as shown in Figure 3. The Right-Leg Drive (DRL) is located at the left arm position, and is used to remove the common noise recorded from the body.

For the duration of this experiment, an inexpensive open source amplifier, OpenEEG, was used to record the ECG signals. OpenEEG was chosen as it is a very basic setup and thus it would be most useful to show the results in conjunction with this system to prove the system will work with all ECG amplifiers. Each system consists of 2 possible input channels, each consisting of a positive and negative input along with the DRL. Each input has a gain of 7812 (38.9dB). Measurement resolution of the A/D converter is 10-bit and has a input voltage resolution of $0.5 \mu \mathrm{V}$. The output of the system is connected to a HUMUSOFT ${ }^{T M}$ card and the data is recorded using MATLAB ${ }^{T M} \mathrm{R} 2008 \mathrm{~b}$. An example of the recorded ECG is shown in Figure 1.

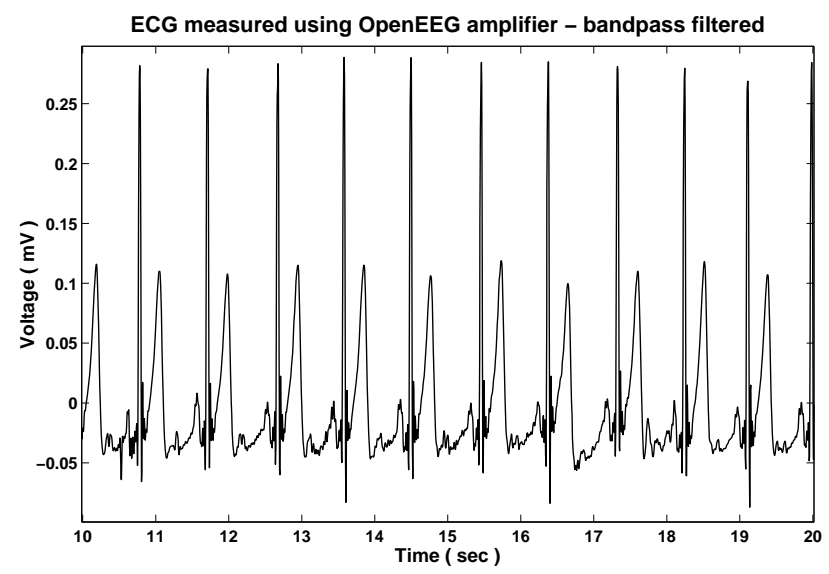

Figure 1. Bandpass filtered ECG signal

For the purposes of this experiment, only one of the amplifiers two channels was required due to the use of the 3-Lead ECG setup. 


\subsection{Motion Artifact}

The ECG amplifier, using two recording electrodes, detects the voltage difference across the chest which arises due to the contractions of the heart. As the human torso changes shape, due to movement, the path between the two recording electrodes is altered. As the medium in which the signal is being recorded changes, the recorded signal will itself encounter some distortion. This adjustment of the original path evokes a disruption to the clean ECG recordings and is known as motion artifact.

A second source of artifact generated externally from the body is due to the movement of the skin/electrode contact. The most effective way to combat electrical changes due to fluctuations in the electrode/skin interface properties is through prevention - preparation of the skin to reduce its impedance/Piezoelectric contribution to the signal [9] and also through the reduction in overall movement of the subject for the duration of the monitoring [10].

The electrodes, in contact with the skin through the conductive gel, are used to measure the biopotentials of the body. These biopotentials are electrical potentials created by ionic currents. These potentials are carried to the body surface, by conductivity, where they can be measured. The ionic current, involving $\mathrm{Na}^{+}, \mathrm{Ca}^{+}$and $\mathrm{Cl}^{-}$ions, has to be transformed into electron current so as to be read by the electrode. The outer skin layer has a dry dielectric which impairs the transfer from ions to electrons in the electrode. The electrolyte gel is used to reduce the influence of the skin on the impedance by moisturising its dry outer layer and making it more ion-conductive [11]. In addition to the impedance of the skin, the electrical transducer comprises the resistance of the electrolytic gel and the double layer at the electrode-electrolyte interface. There is also the half-cell potentials caused by the different energies of the electrode, electrolyte and skin. The equivalent circuit of an electrode skin interface[12] can be seen in Figure 2 with labels explained in Table 1.

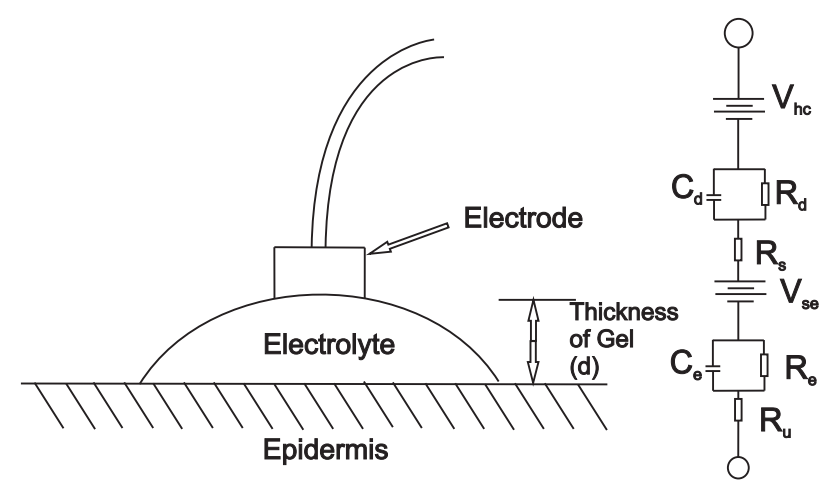

Figure 2. Electrode skin interface with equivalent circuit

Thus, as the distance between the electrode and the epidermis (marked 'd' in Figure 2) varies, so does the resultant equivalent resistance and conductance values. With this change in resistance and conductance of the system, a different output will be recorded by the electrodes. This illustrates that movement of the electrode causes an artifact to be seen on the recorded ECG.

Table 1. Description of Labels in Figure 2

\begin{tabular}{|l||l|}
\hline Label & Description \\
\hline \hline Vhe & Half Cell potential associated with distribution of \\
& charge between Electrode and Electrolyte \\
Cd & Capacitance at Electrode and Electrolyte interface \\
Rd & Resistance at Electrode and Electrolyte interface \\
Rs & Model of resistance in Electrolyte \\
Vse & Half Cell potential associated with distribution of \\
& charge between Electrolyte and Epidermis \\
Ce & Capacitance at Electrolyte and Epidermis interface \\
Re & Resistance at Electrolyte and Epidermis interface \\
Ru & Model of resistance in Epidermis \\
\hline
\end{tabular}

\subsection{Accelerometer System}

The measurement devices used in this system are Analog Devices accelerometers (ADXL203). These accelerometers are capable of measuring both static (gravitational) and dynamic (vibration) dual axis acceleration. The sensor is a low power $\left(700 \mu \mathrm{A}\right.$ at $\left.\mathrm{V}_{s}=5 \mathrm{~V}\right)$ system and has a measurement range of $\pm 1.7 \mathrm{~g}$ with an accuracy of $1 \mathrm{~m} \mathrm{~g}$ at $60 \mathrm{~Hz}$.

The sensor is a polysilicon structure built on top of the base silicon wafer. Polysilicon springs suspend the structure over the surface of the wafer and provide a resistance against acceleration forces. Deflection of this structure is measured using a differential capacitor that consists of independent fixed plates and plates attached to the moving mass. Acceleration deflects the beam and unbalances the differential capacitor, resulting in an output square wave whose amplitude is proportional to acceleration. Phasesensitive demodulation techniques are then used to rectify the signal and determine the direction of the acceleration [13].

For the purpose of this paper we use the Analog Devices ADXL203 accelerometer as a single axis measurement system. Reasons for this are two-fold. Firstly, with an accuracy of $\pm 1 \mathrm{mg}$, and the unlikely situation of the movement being directly orthogonal to the recording axis, movement in any given direction will have an effect on the dominant axis, and thus will remain detectable. Secondly, this setup will show that the system works at the most basic level. Future tests will take advantage of triaxial accelerometers and thus the overall result will become more accurate and robust.

The individual accelerometer outputs are initially biased to remove the gravitational component of the signal, as this information is not required for this paper. The remaining kinematic signal is then amplified and filtered before being passed, using the HUMUSOFT $612^{T M}$ card, into 
MATLAB ${ }^{T M}$ for signal processing. The system is sampled at $200 \mathrm{~Hz}$.

\subsection{Sensor Position}

Placement of sensors is a key issue for detecting motion artifact. As stated previously, motion artifact arises due to movement of the body as well as movement of the electrodes with respect to the skin surface. This movement of the body must then be detected.

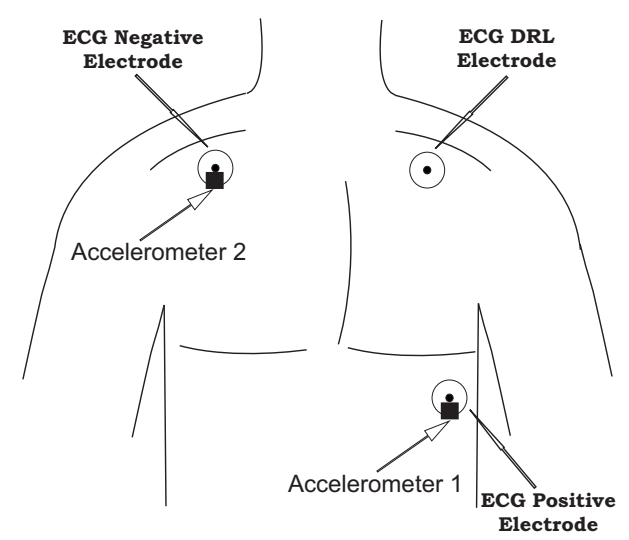

Figure 3. Accelerometer Placement for cross chest movement detection

Figure 3 shows the proposed setup of the accelerometer system for recording the differential movement between the positive and negative recording electrodes. As stated previously, movement of the body causes motion artifact to be observed on the recorded ECG. With the accelerometers positioned as shown, the movement of the electrodes with respect to each other can be found.

The tests undertaken by the subject consisted of 60 second sessions. For the initial 20 seconds, the subject was asked to move their body in any direction they desired, but were asked to try and keep their torso aligned so as to prevent differential movement of the electrodes as much as possible. During the second 20 second period the subject was asked to move their body in any direction or movement they desired, focusing on movement of the torso, so as to cause differential movement. For the final 20 seconds, the subject was asked to remain still such that the minimal amount movement would be detected. These three epochs can clearly been seen in both accelerometer outputs in Figure 4.

\subsection{Data Collection System}

Data from the amplifier circuit and both accelerometer systems are collected on a local PC using a HUMUSOFT
$612^{T M}$ card and are read using the Simulink toolbox in MATLAB ${ }^{T M}$ where it is saved for future processing.

Removal of the baseline wander is required in order to minimise changes in beat morphology that do not have cardiac origin, including respiration. It is known that over $99 \%$ of adults have a mean resting heart rate greater then 44 beats/min [14]. A low-frequency amplitude response down to $0.67 \mathrm{~Hz}$ is necessary to reproduce heart rate frequencies in such a range. It is also recommended that the amplitude response should be flat to within $0.5 \mathrm{~dB}$ over a range of $1.0-30 \mathrm{~Hz}$; the $3 \mathrm{~dB}$ points should be less than or equal to $0.67 \mathrm{~Hz}$ and greater than or equal to $150 \mathrm{~Hz}$ [15]. A bandpass filter is implemented to remove the outlying undesired frequencies.

A notch filter is also required in the system to remove the unwanted artifacts added by the interference from power-lines $(50 \mathrm{~Hz})$ [16]. A sample of ECG having been bandpass and notch filtered can be seen in Figure 1.

To represent the differential movement of the accelerometers which implies movement of one recording electrode with respect to the other, the two accelerometer outputs are subtracted from one another. This resulting signal corresponds to the differential acceleration between the two source outputs.

\subsection{Signal Analysis}

The absolute value of this differential signal is calculated to show the difference between the absolute accelerations of the two electrodes. An empirical threshold value is calculated using the standard deviation of the signal. For the duration of these experiments, a threshold value of three times the standard deviation of the differential signal was used. This value was chosen through experimentation. It was found that the majority of differential movements occurred outside these limits. An example of the accelerometer outputs, differential accelerometer signal and threshold value are shown in Figure 4. If the differential signal has sample points above the threshold line, substantial differential movement is assumed to have occurred at that point.

\section{Results}

Tests were undertaken by a subject to show that differential movement of the recording electrodes has a greater effect on the artifact embedded on the desired ECG then overall subject movement. The subject was asked to complete three 60 second sessions, involving different movements as explained in section 2.4 .

Once the amplifier and accelerometer data was collected from the experiments the signal processing was undertaken. After the differential acceleration and threshold value were calculated, the signal quality could be determined. All differential acceleration sample points, whose acceleration was greater than the set threshold, were assigned as detected motion. Finally, a signal quality level 

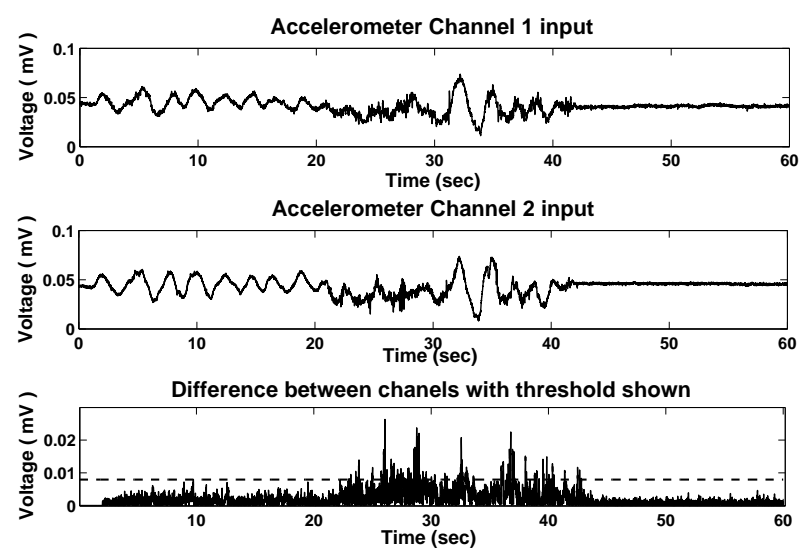

Figure 4. Accelerometer outputs for channel 1 and 2. Normalised differential signal with threshold value illustrated

was created. This algorithm calculated the number of movements that were detected over a 1 second period, centred at the current sample. The current sample was then assigned a value between 0 and 1 indicating the number of movements were detected in that one second epoch. A value of 1 was given to a detected clean signal (ie no detected movements) with zero showing all surrounding samples had also detected movement.

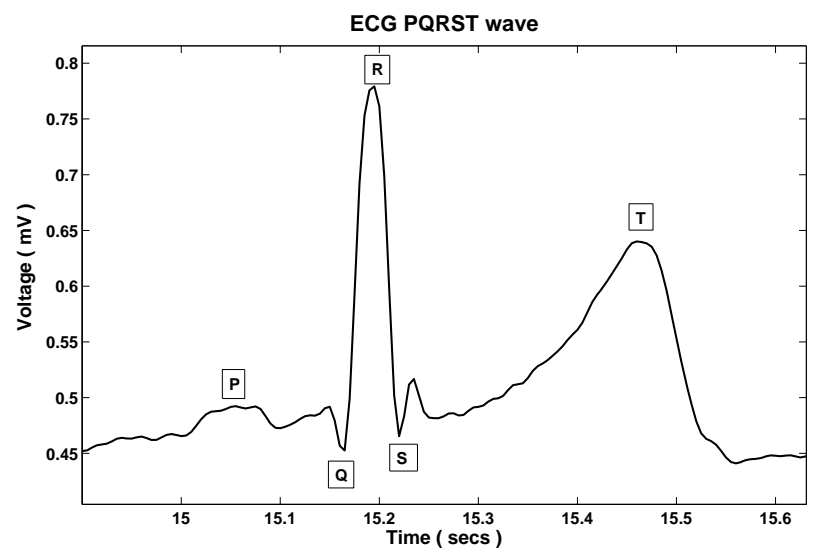

Figure 5. Section of clean ECG with clear PQRST positions shown

For skilled technicians reviewing the outputted ECG the most fundamental necessity is the ability to observe clean signals consisting of individual P QRS and T waves. These waves can be seen in Figure 5 taken from the test ECG. The presence, or indeed absence, of these waves in clean ECG can inform a skilled technician of a number of different ailments. Therefore the inability to distinguish these waves, due to motion artifact, causes a dilemma. In Figure 6 a number of the waves cannot be distinguished within the heartbeats in the ECG signal and are marked with an ' $X$ '. It can be seen that all occasions in which the waves of the ECG signal appear to be undiscernible, the signal quality level also shows a decrease.
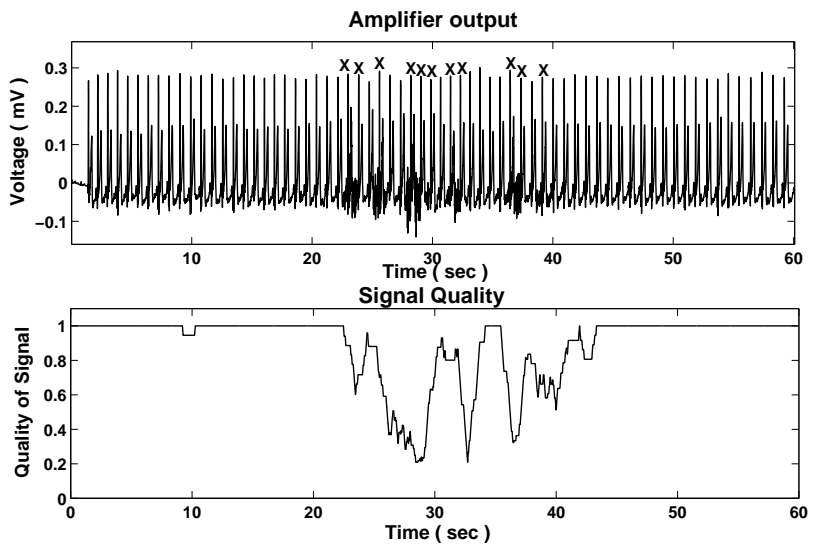

Figure 6. Unclear PQRST waves marked manually. Signal quality output from algorithm

Figure 7 shows a section of the signal based around the transition between the full body movement epoch, encompassing the initial 20 seconds, and the torso movement epoch. At twenty seconds the subject was asked to begin to move their torso creating differential movement between the two electrodes. It can be clearly seen that as the accelerometers pick up this differential movement, thus creating the signal quality level, the ECG also detects the movement, causing motion artifact.
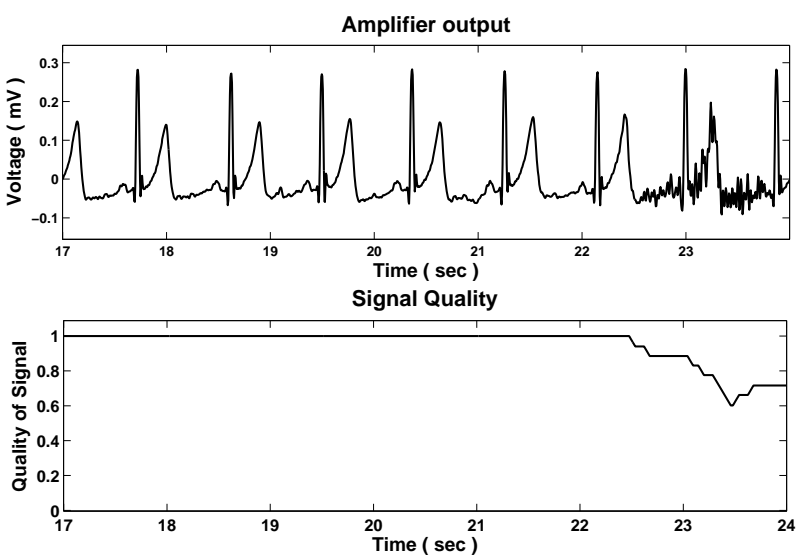

Figure 7. Section of ECG during full body movement (Up to 20 seconds). Full Torso movement (after 20 seconds)

Although for the duration of the second 20 second epoch the subject was continually in motion, it can again be seen in Figure 8 that it is only where differential movement occurred that the ECG embeds the motion artifact.

Figure 9 shows the comparison between the QOS resulting from the use of the differential accelerometer setup (seen previously in Figure 6) and a naive QOS method using a single accelerometer. For the latter a simple algorithm is implemented to detect movement using the output 

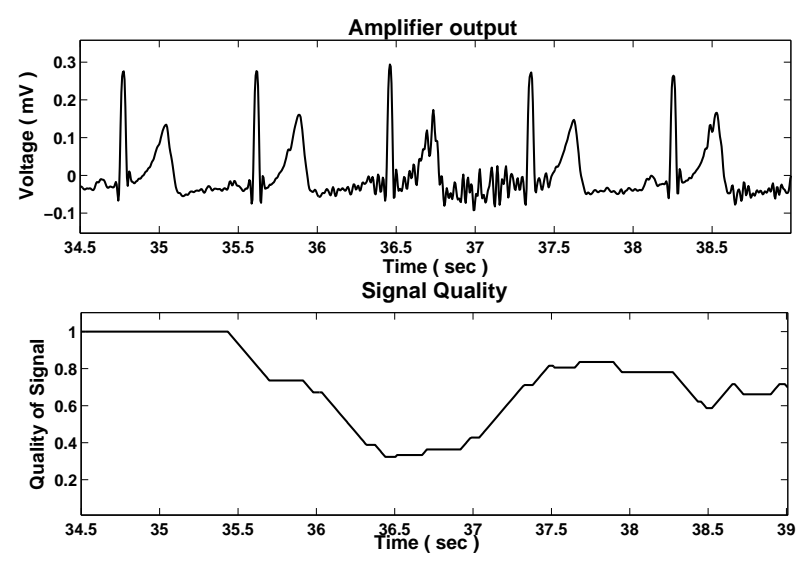

Figure 8. Section of ECG during Torso body movement

of a single accelerometer as a reference. The same QOS algorithm used previously is then executed on the detected movement from this single accelerometer. With the use of only a single accelerometer as a motion reference, there is a much lower QOS metric over the recorded signal. Using differential accelerometers $34.4 \%$ of the recorded ECG signal is determined to be contaminated with motion artifact while using only a single accelerometer this contamination level rises to $65.7 \%$.
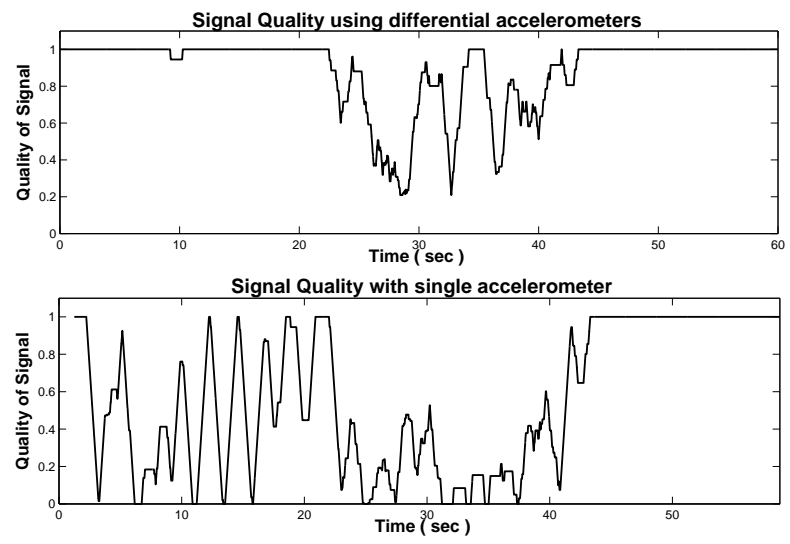

Figure 9. Comparison between differential accelerometer QOS and QOS implemented from single accelerometer

\section{Discussion and Conclusion}

To date, all ECG systems which have a signal quality level attributed to their output, do so with use of one or more accelerometers. These accelerometers are currently only detecting overall movement of the test subjects. This implies that, as the subjects body position shifts, or alternates in any direction, the calculated signal quality level deteriorates.

In this paper, an accelerometer system was implemented which detects the differential acceleration between the two recording electrodes, thus detecting when one electrode is moving in relation to the other. Using this system it has been demonstrated that all ranges of body movement do not necessarily cause motion artifact to be seen on the recorded ECG. It can be observed in Figure 6 that when the subject is in constant motion, but with no differential movement of the electrodes, this motion has no ill effect on the ECG. However, in the areas where differential movement of the electrodes is introduced (approximately between 2040 seconds) there is a noticeable decline in the quality of the signal (Figure 8).

Previous methods for applying a QOS metric, with the use of only a single sensor to detect movement of the body, results in a lower QOS over the recorded signal (Figure 9). In this example there is almost a doubling in the amount of data labeled as having some contamination from motion artifact. This results in some clean ECG signal being wrongly labeled, which may lead to it being ignored in subsequent analysis of the recording.

The use of this new QOS technique as a method for determining signal quality will decrease the volume of wrongly labeled source data and by doing so will provide any technician studying the stress ECG signal with a more accurate evaluation of the areas where motion artifact is likely to appear on the signal. Due to the simplicity of the system and algorithms, it will allow them to be easily embedded into systems currently used for in-home monitoring.

Future work to be completed in this area includes the use of triaxial accelerometers, to improve the accuracy of the system. The author also plans to investigate the effect of electrode movement, with respect to the skin, on the motion artifact. This shall be explored by placing one accelerometer on the recording electrode while another is placed on the skin in close vicinity. By detecting differential movement between the two accelerometers the system is detecting changes in d (Figure 2), the distance between the electrode and epidermis, thus showing when motion artifact is likely to have occurred.

\section{Acknowledgements}

This research is funded by the Irish Research Council for Science, Engineering and Technology (IRCSET)

\section{References}

[1] A. Gautam, Young-Dong Lee, and Wan-Young Chung. Noise cancellation of electrocardiogram signal measured by wearable USN node. In Multisensor Fusion and Integration for Intelligent Systems, 2008. IEEE International Conference on, pages 154-157, Aug. 2008.

[2] N.V. Thakor and Y.-S. Zhu. Applications of adaptive filtering to ECG analysis: noise cancellation and ar- 
rhythmia detection. Biomedical Engineering, IEEE Transactions on, 38(8):785-794, Aug. 1991.

[3] D.A. Tong, K.A. Bartels, and K.S. Honeyager. Adaptive reduction of motion artifact in the electrocardiogram. In 24th Annual EMBS/BMES Conference, 2002. Proceedings of the Second Joint, volume 2, pages 1403-1404, 2002.

[4] M. Mathie, J. Coster, A. Lovell, and B G Celler. Detection of daily physical activities using a triaxial accelerometer. Medical and Biological Engineering and Computing, volume 41:296-301, 2003.

[5] M.A.D. Raya and L.G. Sison. Adaptive noise cancelling of motion artifact in stress ECG signals using accelerometer. In 24th Annual EMBS/BMES Conference, 2002. Proceedings of the Second Joint, volume 2, pages 1756-1757, 2002.

[6] J. Muhlsteff, O. Such, R. Schmidt, M. Perkuhn, H. Reiter, J. Lauter, J. Thijs, G. Musch, and M. Harris. Wearable approach for continuous ECG - and activity patient-monitoring. In IEMBS 2004. 26th Annual International Conference of the IEEE, volume 1, pages 2184-2187, Sept. 2004.

[7] J. Healey and B. Logan. Wearable wellness monitoring using ECG and accelerometer data. In Wearable Computers, 2005. Proceedings. Ninth IEEE International Symposium on, pages 220-221, Oct. 2005.

[8] Y. Kishimoto, Y. Kutsuna, and K. Oguri. Detecting Motion Artifact ECG Noise During Sleeping by Means of a Tri-axis Accelerometer. In EMBS 2007. 29th Annual International Conference of the IEEE, pages 2669-2672, Aug. 2007.

[9] J G. Burbank, D P. Webster. Reducing skin potential motion artefact by skin abrasion. Medical and Biological Engineering and Computing, volume 16:31-38, 1978.

[10] K. Kearney, C. Thomas, and E. McAdams. Quantification of Motion Artifact in ECG Electrode Design. In EMBS 2007. 29th Annual International Conference of the IEEE, pages 1533-1536, Aug. 2007.

[11] A. Karilainen and J. Hansen, S. Muller. Dry and capacitive electrodes for long-term ECG-monitoring. 8 th Annual Workshop on Semiconductor Advances, pages 155-161, 2005.

[12] J. G. Webster. Medical Instrumentation, Application and Design. Wiley, 1998.

[13] Inc Analog Devices. Precision $1.7 \mathrm{~g}$ Single-/DualAxis iMEMS Accelerometer. Analog Devices, Inc, One Technology Way, P.O. Box 9106, Norwood, MA 02062-9106, U.S.A., 2006.
[14] E. Simonson. Differentiation between normal and abnornal in electrocardiography. Proceedings of the Royal Society of Medicaine, 55(10), 1962.

[15] JJ Bailey, AS Berson, Jr Garson, A, LG Horan, PW Macfarlane, DW Mortara, and C Zywietz. Recommendations for standardization and specifications in automated electrocardiography: bandwidth and digital signal processing. Circulation, 81(2):730-739, 1990 .

[16] A. V. Oppenheim and R. W. Schafer with J.R Buck. Discrete-Time Signal Processing. Prentice-Hall, 1989. 